\title{
Assessment of cardiac dyssynchrony: a comparison of velocity encoded imaging and feature tracking analysis
}

\author{
Daniel Kuetting ${ }^{*}$, Jonas Doerner, Alois M Sprinkart, Claas P Naehle, Hans H Schild, Daniel Thomas \\ From 16th Annual SCMR Scientific Sessions \\ San Francisco, CA, USA. 31 January - 3 February 2013
}

\section{Background}

Cardiac dyssynchrony can be found in up to $99 \%$ of patients suffering from chronic heart failure. MRI VENC (velocity encoded imaging), along with TDI, have been shown to accurately assess myocardial dyssynchrony. Long acquisition times of VENC and a high inter-examiner variability of TDI have prohibited a broad clinical application of these modalities thus far. Recently a new method utilizing feature tracking was introduced which allows to analyze strain, respectively velocities, in standard SSFP cine images.

This study sought to assess the feasibility of evaluating dyssynchrony in standard SSFP cine images with the use of feature tracking analysis.

\section{Methods}

Velocity encoded MRI was performed in 2 groups, 13 healthy control subjects (group A) and 6 dyssynchronous patients pre- CRT (cardiac resynchronization therapy) (group B). Additionally, high temporal resolution SSFP cine images were acquired. Quantitative analysis of dyssynchrony in VENC encoded MR-images was performed using Segment 1.9 software package (Medviso AB). Venc imaging was performed in the four-chamber orientation. Velocity was encoded with a velocity sensitivity of $20 \mathrm{~cm} /$ s. Additionally SSFP-cine images with the same temporal resolution were acquired in the four chamber orientation.
Velocity was measured in the basal segments of the lateral free wall and the septum. The analysis of dyssynchrony in SSFP cine-images was performed with 2D CPA MR (TomTec Imaging Systems, Germany).

\section{Results}

Intraventricular dyssynchrony was not observed in group A (mean dyssynchrony $12.3 \pm 9.6 \mathrm{~ms}$ using VENC analysis, mean dyssynchrony $10.6 \pm 9.6 \mathrm{~ms}$ on feature tracking $(\mathrm{p}=\mathrm{NS}))$. Group B displayed a mean intraventricular dyssynchrony of $77.9 \pm 29.5 \mathrm{~ms}$ with VENC analysis, $74.8 \pm$ $26.5 \mathrm{~ms}$ with feature tracking respectively $(\mathrm{p}=\mathrm{NS})$. The feature tracking method reliably discriminated between dyssynchrony and physiologically timed contraction in all cases. A good correlation (Pearson 0,9673 $\mathrm{P}<0,0001$ ) and agreement (mean difference 2.7 with a $95 \% \mathrm{CI}$ of $-1,7875$ to 7,5057 ) were found between VENC MR imaging and feature tracking.

\section{Conclusions}

Preliminary results suggest that feature tracking analysis of standard SSFP-cine images can yield equivalent information on intraventricular dyssynchrony to VENC imaging. This would permit an assessment of dyssynchrony in a clinical setting without having to acquire time consuming VENC images. Future studies are needed to further validate these findings. 


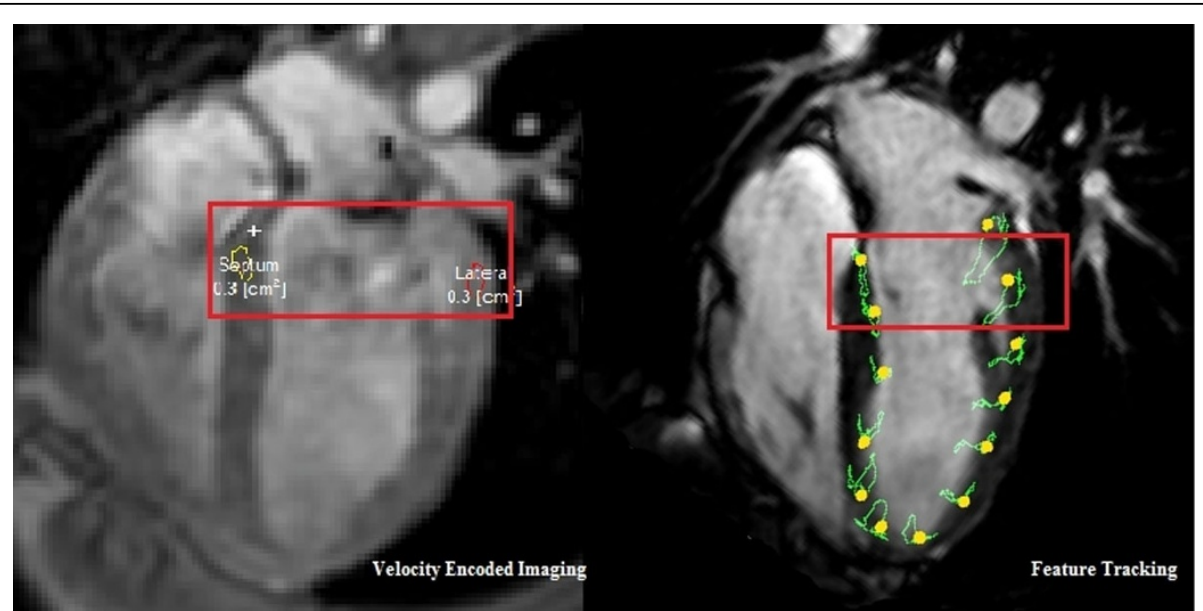

Figure 1 Defined regions of velocity measurement. (left image: Velocity Encoded Imaging; right image: Feature Tracking)

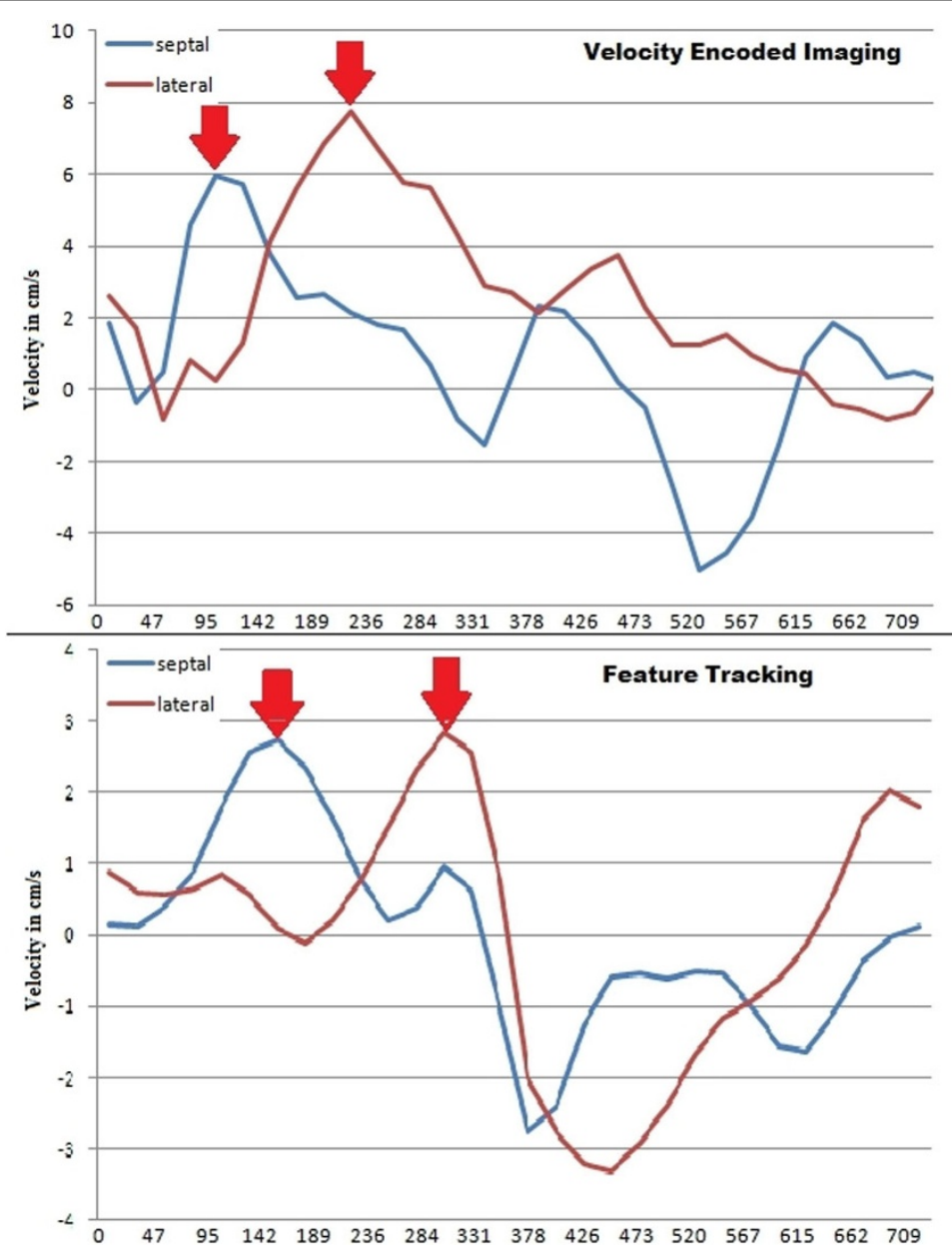

Figure 2 Results of Velocity Encoded Imaging (upper graph) and Feature Tracking (lower graph) analysis of a dyssynchronous patient displaying extensive septal-to-lateral-delay. 


\section{Funding}

There was no funding source for this study.

Published: 30 January 2013

doi:10.1186/1532-429X-15-S1-M11

Cite this article as: Kuetting et al:: Assessment of cardiac dyssynchrony:

a comparison of velocity encoded imaging and feature tracking

analysis. Journal of Cardiovascular Magnetic Resonance 2013

15(Suppl 1):M11.

Submit your next manuscript to BioMed Central and take full advantage of:

- Convenient online submission

- Thorough peer review

- No space constraints or color figure charges

- Immediate publication on acceptance

- Inclusion in PubMed, CAS, Scopus and Google Scholar

- Research which is freely available for redistribution 